\title{
EMOÇÕES ACADÊMICAS, AUTORREGULAÇÃO E SEU IMPACTO SOBRE MOTIVAÇÃO E APRENDIZAGEM ${ }^{1}$
}

\author{
ACADEMIC EMOTIONS, SELF-REGULATION, AND THEIR IMPACT ON \\ MOTIVATION AND ACHIEVEMENT
}

\section{EMOCIONES ACADÉMICAS, REGULACIÓN, Y SU IMPACTO SOBRE MOTIVACIÓN Y DESEMPEÑO}

José Aloyseo Bzuneck ${ }^{2}$

\section{RESUMO}

O texto é uma revisão da literatura mais recente sobre emoções acadêmicas e sua autorregulação. Com base em dados de pesquisa, são descritas emoções positivas e negativas, suas origens e seus efeitos sobre a motivação e o desempenho, à luz das teorias de atribuição de causalidade, de Weiner e de controle-valorização, de Pekrun. Foram selecionadas as emoções negativas de vergonha, tédio e ansiedade, mais frequentemente focalizadas nos estudos, sobre as quais foram descritas diversas estratégias de autorregulação que os alunos podem usar nos contextos de aprendizagem. Foi discutida a relevância da teoria social cognitiva para explicar os processos de autorregulação, bem como para ajudar alunos a se tornarem capazes de regular emoções negativas. Por último, foram dadas sugestões de pesquisa futuras em nosso meio.

PALAVRAS-CHAVE: Emoções acadêmicas. Autorregulação emocional. Teorias sobre emoções. Motivação acadêmica.

\begin{abstract}
The present paper reviews recent literature about academic emotions and their regulation. Research-based data about positive and negative emotions, their sources and effects on motivation and achievement were described, on the light of Weiner's Causal Attribution Theory and Pekrun's Control-value Theory. Regarding negative emotions of shame, boredom, and anxiety, more often examined in the literature, several selfregulation strategies were related that students can use in achievement contexts. The relevance of the Social Cognitive Theory was iscussed as a reference aimed at explaining the process of emotional self-regulation and at helping students to develop self-regulatory skills. Last, suggestions for future studies in the area in our country were given.
\end{abstract}

KEY WORDS: Academic emotions. Emotional Self-Regulation. Theories about Emotions. Academic Motivation.

\section{RESUMEN}

El texto constituye una revisión de la literatura reciente acerca de emociones académicas y de su regulación. Emociones positivas y negativas son descritas según dados de investigaciones hasta el presente, sus orígenes y sus efectos sobre la motivación y el desempeño. La teoría de atribución de causalidad, de Weiner y la teoría de control-valorización, de Pekrun son los referenciales más empleados en los estudios. En relación específica a las emociones de ansiedad, aburrimiento y vergüenza, que son más frecuentemente examinadas en la literatura, fueran relatadas las estrategias de regulación utilizadas por los estudiantes en los contextos de aprendizaje. La relevancia de la Teoría Social Cognitiva fue discutida como un referencial para explicar los procesos de regulación emocional y para ayudar los alumnos a desarrollar habilidades de su regulación. Por último, fueran presentadas sugestiones para nuevos avanzos en los estudios sobre el tema.

\footnotetext{
${ }^{1}$ Este artigo tem como base a Conferência proferida pelo autor no II Seminário Internacional Teoria Social Cognitiva em Debate. Rio Claro-SP: UNESP, 2017.

${ }^{2}$ Doutor em Psicologia Escolar e do Desenvolvimento Humano - Universidade de São Paulo (USP) - São Paulo, SP - Brasil. Professor titular - Universidade Estadual de Londrina (UEL) - Londrina, PR - Brasil. E-mail: bzuneck@sercomtel.com.br
}

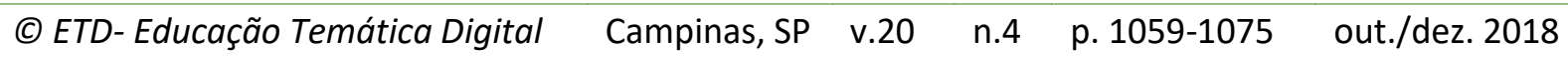


PALABRAS CLAVE: Emociones académicas. Regulación de las emociones. Teorías sobre emociones. Motivación académica.

\section{INTRODUÇÃO}

A autorregulação de emoções nos contextos educativos é uma atividade pessoal a ser exercida em concomitância com a autorregulação da aprendizagem e da motivação, necessária quando assomarem emoções prejudiciais às aprendizagens e ao rendimento escolar (BANDURA, 2006; PINTRICH, 2004; ZIMMERMAN, 2013). Como alunos irão regular tais emoções será, portanto, o foco do presente artigo. Mas, o que são emoções e, mais especificamente, de quais emoções estamos tratando?

Aqui será adotado o conceito proposto por Scherer (2009), segundo o qual emoção é um processo emergente e dinâmico que tem como base uma avaliação subjetiva de eventos significativos. De acordo com essa conceituação, adotada por autores como Op't Eynde e Turner (2006) e Pekrun et al. (2011), faz parte de todo processo emocional um componente cognitivo, que consiste na interpretação e avaliação de objetos, situações ou pessoas, às quais se associam reações neurofisiológicas. Além disso, emoções se revelam em expressões motoras, facilmente identificáveis, por exemplo, nas emoções da raiva ou de medo, e têm potencial para ação. Quanto à valência, temos emoções positivas ou negativas, sendo que ambas variam em intensidade, duração e especificidade situacional.

Emoções estão presentes em todas as situações humanas, que incluem o exercício profissional, as relações interpessoais, os esportes, a participação política e a religiosidade. No presente artigo, serão consideradas apenas as emoções próprias do contexto educativo, que Pekrun (2006) denominou emoções acadêmicas, ou de desempenho (academic emotions; achievement emotions). São emoções dos alunos que surgem durante as aulas, ou quando estudam, quando prestam uma prova, ou quando fazem o dever de casa e, por fim, em função de uma avaliação, que se segue aos desempenhos apresentados (PEKRUN, 2006).

No contexto das aprendizagens escolares, têm-se examinado emoções positivas, como satisfação, orgulho, esperança, alívio e, entre as negativas, ansiedade, medo, desesperança, vergonha e tédio (por ex., AHMED et al., 2013; MEGA; RONCONI; DEBENI, 2014; PEKRUN et al., 2011). As emoções acadêmicas têm a característica de poderem flutuar conforme as situações ou alterar-se pela simples passagem do tempo. Na escola, elas podem desenvolver-se ao longo de um ano letivo, manter-se estáveis ou atenuar-se. Alguns estudos examinaram essas tendências (por ex., AHMED et al., 2013; VIERHAUS et al., 2016). Vierhaus et al. constataram que a satisfação declina na passagem para a segunda fase do ensino fundamental, enquanto que, nessa situação, o tédio tende a aparecer com mais frequência, isto é, as trajetórias de satisfação e de tédio, na vida escolar, aprecem como invertidas. 
Teorias sobre as origens próximas de emoções acadêmicas

Duas teorias contemporâneas, mais frequentemente referidas na literatura, focalizaram explicitamente os processos intrapessoais em virtude dos quais surgem emoções nos contextos de realização acadêmica: a Teoria das atribuições de causalidade, de B. Weiner, e a Teoria controle-valorização, de R. Pekrun. Ambas as teorias têm como foco o componente cognitivo básico das emoções, que consiste na avaliação de uma dada situação.

Weiner (1985) incorporou as emoções em seu modelo motivacional, fundamentado nas atribuições causais. Emoções - positivas ou negativas - resultam tanto do próprio evento de sucesso ou de fracasso, como das atribuições dadas a tais eventos. No caso de sucesso percebido, a pessoa experimentará satisfação e felicidade e, nos casos de fracasso ou resultado negativo, tenderá a sentir frustração, tristeza, infelicidade.

Adicionalmente, segundo essa teoria, o aspecto mais importante é que emoções específicas surgirão em decorrência das causas às quais for atribuido o evento de sucesso ou de fracasso. Assim, orgulho e autoestima positiva serão experimentados nos casos em que o sucesso for creditado a causas internas ao próprio indivíduo, tais como inteligência ou esforço, mas a pessoa terá sentimento de culpa quando o fracasso for atribuído à falta de empenho. $O$ aluno sentirá irritação, quando o fracasso for atribuído a fatores incontroláveis e externos, como prova excessivamente difícil, mas será acometido pela vergonha, desesperança ou resignação, quando o fracasso for atribuído a fatores internos não controláveis, como falta de capacidade. Todas essas emoções têm, ademais, um papel motivacional, isto é, trazem em si um potencial para a ação.

Pela teoria de Controle-valorização, desenvolvida por Pekrun (2006), toda emoção no contexto das aprendizagens escolares resulta da combinação de um duplo julgamento por parte do aluno - de controle e de valorização. Por exemplo, satisfação surgirá quando o aluno perceber que sua aprendizagem está sob seu controle e que tem valor ou importância pessoal. Controle por parte do aluno refere-se à expectativa de que, com seu esforço e persistência, terá resultados positivos. Mas refere-se também a controle sobre o próprio agir, que é um conceito compatível com o de autoeficácia. Já valorização corresponde à percepção de que o resultado à vista tem importância pessoal, por exemplo, para sua autorrealização, ou porque tem valor instrumental em relação a outros objetivos.

O impacto das emoções no contexto escolar

A produção científica na área tem fornecido evidências de que as emoções acadêmicas têm importância crítica para o desenvolvimento cognitivo, para a motivação e a aprendizagem autorregulada e, por fim, para o desempenho. Assim, emoções positivas sempre apareceram relacionadas significativamente com desempenho acadêmico. No estudo de Pekrun et al. (2002), por exemplo, descobriram-se relações positivas e significativas entre 
satisfação e esperança com a utilização de estratégias de elaboração, organização, metacognição e pensamento crítico. O estudo de Mega et al. (2014), por sua vez, mediante a modelagem das equações estruturais, mostrou que o conjunto de emoções positivas influencia, de modo direto, a autorregulação e os fatores motivacionais de autoeficácia e metas de realização, sendo que, somente por meio dessas variáveis, as emoções afetam o desempenho final.

De acordo com Bandura (2006) e Frederickson (2001), entre outros, tais associações se explicam pelo fato de que, quando ativadas, as emoções positivas tendem a expandir o repertório de ações derivadas de pensamentos, isto é, elas abrem a mente e, assim, desafios são enfrentados com mais disposição. Neurologistas (por ex., ASHBY et al., 1999; DAl; STERNBERG, 2004) acrescentaram que o afeto positivo está associado a níveis mais elevados de dopamina, um neurotransmissor que produz efeitos positivos sobre vários aspectos do processamento cognitivo. De resto, segundo Linnenbrink-Garcia and Pekrun (2011), tem-se intensificado a investigação sobre as bases neurológicas das emoções e sua associação com outras formas do funcionamento neurológico.

Efeitos opostos aos das emoções positivas têm sido amplamente documentados na literatura para as negativas. Como amostra, entre os estudos mais recentes, Mega et al. (2014), com sua análise que aglutinou diversas emoções negativas de estudantes universitários, concluíram que elas têm valor de predição e de influência negativa sobre motivação e aprendizagem autorregulada, com repercussão final no desempenho. Eckert et al. (2016), com amostra similar, descobriram que emoções negativas estavam altamente relacionadas com comportamentos de procrastinação, confirmando outros estudos anteriores, como o de Steel (2007).

$\mathrm{Na}$ literatura da psicologia educacional, têm sido objeto de investigação mais intensa as emoções negativas de raiva, vergonha e tédio, destacando-se a ansiedade. Cada uma delas será brevemente descrita, com a indicação de suas origens e efeitos.

Irritação ou raiva será sentida quando um aluno atribuir seu fracasso no rendimento escolar a fatores incontroláveis e externos a si próprio, tais como prova excessivamente difícil, ou a critérios injustos adotados pelo professor (WEINER, 1985). No amplo estudo de Pekrun et al. (2011), essa emoção apareceu positivamente correlacionada com ansiedade e negativamente com a medida de esforço, tanto na hora de estudo, como em sala de aula e por ocasião de provas. As mesmas relações foram descobertas no referente à emoção de desesperança.

Vergonha ou humilhação, como componente da baixa autoestima, tem origem na atribuição de desempenho pobre ou de fracasso à falta capacidade, associada a uma autopercepção de boa aplicação de esforço. Capacidade é considerada fator interno, estável e não controlável (WEINER, 1985), de modo que a atribuição à sua falta explica o julgamento autodepreciativo. Turner, Husman e Schallert (2002), que adotaram essa mesma explicação, 
apontaram que vergonha, enquanto perdura, costuma produzir efeitos devastadores sobre a motivação e, por fim, sobre o desempenho. Embora resulte da experiência de fracasso e de sua interpretação, numa situação específica, é a pessoa na sua totalidade que se sente humilhada. As mesmas autoras constataram que vergonha tem como fatores desencadeadores baixas crenças de autoeficácia e alta ansiedade numa situação de prova.

Tédio tem sido frequente objeto de estudo na literatura mais recente. É descrita como emoção desagradável, marcada por desinteresse e baixa ativação (VOGEL-WALKUTT et al., 2012) e como um estado de indiferença, languidez e apatia (CSIKSZENTMIHALYI, 1990), comum em sala de aula. No estudo de Nett, Goetz e Hall (2011), estudantes do ensino médio relataram ter experimentado tédio em $58 \%$ do tempo em aulas de matemática. Para Pekrun et al. (2014), apoiados em vasta literatura, tédio está associado a devaneios e à vontade de escapar dessa condição mediante desengajamento, ficando, em consequência disso, reduzidos os recursos cognitivos e motivacionais nas aprendizagens. Além disso, os mesmos autores relataram haver evidências de que o tédio se relaciona negativamente com o nível de capacidade cognitiva dos alunos.

Em vários estudos têm surgido, consistentemente, correlações negativas entre tédio, por um lado, e motivação, uso de estratégias adequadas de aprendizagem e desempenho acadêmico, por outro lado (por ex., NETT, GOETZ; DANIELS, 2010; NETT et al., 2011; PEKRUN et al. 2002; PEKRUN et al., 2014, TZE, DANIELS; KLASSEN, 2016). Salienta-se nesses estudos que, quando entediados, alunos adolescentes raramente adotavam estratégias focalizadas nos problemas e, com mais frequência, revelavam evitação da tarefa, distração mental ou simplesmente uma tolerância passiva dessa condição. Em outras palavras, alunos entediados caracterizavam-se por desperdiçar um tempo precioso, que deveria ser bem investido nas atividades de aprendizagem.

Csikszentmihalyi (1992) sugeriu que o tédio surge quando o aluno se avalia com alto controle em relação à execução de certa tarefa, pelo fato de já tê-la dominado, ou seja, ela não mais aparece como desafiadora. Como explicação alternativa, Pekrun et al. (2014) evocaram a teoria do controle-valorização para explicar que o tédio tem duas raízes: (a) a percepção de baixo controle pessoal na execução da atividade, porém, em função de excessivo controle externo, e (b) a percepção de baixa valorização daquela atividade, que aparece como irrelevante, sem importância pessoal. Dados de pesquisa têm comprovado essas relações (PEKRUN et al., 2014; TANAKA; MURAYAMA, 2014).

Ansiedade é, de longe, a emoção acadêmica negativa mais examinada em pesquisas. Também foi a mais relatada por uma amostra de universitários nas provas (PEKRUN et al., 2002). Tem sido conceituada como um estado de tensão e desconforto, normalmente acompanhada de medo (ERTURAN; JANSEN, 2015; HEMBREE, 1988). Spielberger (1966) havia defendido ser necessário distinguir entre ansiedade-estado e ansiedade-traço, uma distinção mantida até o presente em diversos estudos (por ex., AHMED et al., 2013; FIORAVANTIBASTOS et al., 2011; HARPELL; ANDREWS, 2013; PEKRUN et al., 2002). A primeira é uma 
emoção transitória, provocada por uma situação específica, enquanto que a segunda é uma inclinação ou disposição crônica, que emerge como estado numa grande variedade de situações. Nos contextos de aprendizagem escolar, têm sido identificadas, como particularmente frequentes, ansiedade em ralação à matemática e ansiedade nas situações de prova (por ex., BZUNECK, 1991; CASSADY; JOHNSON, 2002; ERTURAN; JANSEN, 2015; JUSTICIA-GALIANO et al., 2016; VOGELAAR et al., 2017). A ansiedade de prova seria uma expressão da ansiedade-traço, porém, com possível influência adicional de outros fatores, como o contexto da prova, a percepção de ameaça, a autopercepção de competência, padrões pessoais de enfrentamento e de motivação e até habilidades de estudo (ZEIDNER, 2014). Pekrun (2006), entretanto, observou que, devido a experiências anteriores de dificuldades e de pressão em avaliações, certos alunos desenvolvem emoções acadêmicas habitualizadas, como ansiedade, daí resultando certos esquemas mentais procedimentais. $\mathrm{Na}$ sequência, a simples ocorrência de nova prova ou a situação de entrar em classe da disciplina em questão, ou até o fato de estudar aquela matéria induzirá o aluno, de modo inconsciente, a evocar aquele esquema mental e sentir ansiedade.

Embora vários autores tenham concluído que meninas figuram mais que meninos entre os portadores de ansiedade-traço, pela recente revisão de Reali et al. (2016), constatouse em alguns estudos não haver diferença significativa em função de gênero. De resto, ansiedade-traço tem, como antecedentes, fatores tanto biológicos como ambientais (ZEIDNER, 2014). Quando se consideram os estados de ansiedade que surgem por ocasião de provas, importa muito considerar seu grau de intensidade. Assim, essa emoção pode estar ativada ou em grau mínimo, ou em grau moderado, ou em alto grau, configurando-se, neste último caso, alta ansiedade. Quando seu grau for muito baixo, não há predisposição para a ação, ou seja, ela não contribui para o engajamento, dada a reduzida ativação geral, que teria como origem uma percepção de pouco desafio ou de irrelevância da tarefa. No extremo oposto, alta ansiedade será normalmente prejudicial. Já níveis moderados de ansiedade ou tensão não somente não prejudicam como até favorecem o engajamento nas tarefas (CASSADY; JOHNSON, 2002).

$\mathrm{Na}$ literatura tem sido comprovado, consistentemente, que alta ansiedade constitui um grande problema para muitos alunos, em todos os níveis de escolaridade, por produzir um efeito debilitante sobre os processos de aprendizagem e sobre o rendimento. Por exemplo, alta ansiedade mantida ao longo de uma prova é responsável por um rendimento abaixo do esperado (por ex., BZUNECK, 1991; CASSADY; JOHNSON (2002); HEMBREE, 1988; JACOBS; GROSS, 2014; JUSTICIA-GALIANO et al., 2016; PEKRUN et al. ,2011; REALI et al., 2016; ZEIDNER, 2014). Mas, além de afetar negativamente o desempenho em provas, alta ansiedade prejudica o próprio processamento das informações, já antes, por ocasião dos estudos de preparação (CASSADY; JOHNSON, 2002). Zeidner (2014) elencou e descreveu 15 fases e aspectos da aprendizagem em que alta ansiedade produz tais efeitos perversos. É

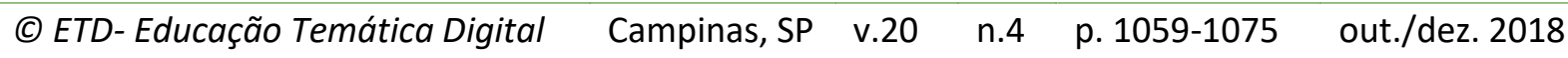


razoável a inferência de que com esses efeitos é compatível o resultado do exame PISA de 2012 (OECD, 2013) segundo o qual, nos países em que foi mais alta a ansiedade em relação à matemática, também foi mais baixo o escore médio nessa disciplina.

A explicação do efeito debilitante desse estado emocional, tradicionalmente adotada também por vários autores (por ex., AHMED ET AL., 2013; CASSADY; JOHNSON, 2002; DECUIRGUMBI, AULTMAN; SCHUTZ, 2009; ERTURAN; JANSEN, 2015; ZEIDNER, 2014) tem origem em dados da pesquisa de Liebert e Morris (1967), que concluíram pela presença de dois componentes distintos na alta ansiedade por ocasião de provas: a preocupação e a emotividade. A emotividade representa os sintomas psicofisiológicos, como sudorese, taquicardia etc. A preocupação, que emerge junto com a experiência de emotividade, porém dela distinta, (HEMBREE, 1988; ZEIDNER, 2014), está associada a medo de fracasso e pertence à categoria cognitiva de um pensamento intruso ou diálogo interno que, por exemplo, numa prova, assoma instantânea e involuntariamente na consciência e é persistente. Cassady e Johnson (2002) optaram por denominar esse componente de ansiedade cognitiva de provas. Seu conteúdo consiste de ruminações mentais do aluno sobre si próprio, ou sobre a dificuldade da tarefa, sobre seu desempenho e sobre as consequências.

São exemplos típicos de diálogo interno: "eu deveria ter repassado mais vezes a matéria; desse jeito não vou conseguir mesmo" (preocupação com o desempenho); "ontem eu sabia tudo isso: que é que há comigo?" (Preocupação consigo próprio); "tenho certeza de que todos estão fazendo melhor que eu" (preocupação relacionada aos outros); "preciso de nota, deste jeito vou reprovar" (preocupação com as consequências). Ora, tal diálogo interno espúrio, quer no momento de uma prova, quer antes, em sua preparação, compromete a atenção, que deveria manter-se inteiramente focalizada na tarefa, impedindo, além disso, que a memória de trabalho evoque conhecimentos adquiridos e realize os processamentos necessários (CASSADY; JOHNSON, 2002; ERTURAN; JANSEN, 2015; JUSTICIA-GALIANO et al., 2016).

\section{AUTORREGULAÇÃO DAS EMOÇÕES}

As grandes quantidades de dados fornecidos pelas pesquisas mencionadas levam à conclusão, defendida por autores como Op't Ende e Turner (2006) e Pekrun et al. (2002), de que as emoções acadêmicas não são meros efeitos colaterais, mas fazem parte integral dos próprios processos de aprendizagem e da motivação. Entretanto, um fato básico é que emoções negativas são inevitáveis, já que não é possível impedir que todos os alunos passem por experiências de dificuldade, de fracasso e de frustrações ao longo de sua escolaridade, cuja interpretação dá origem a emoções negativas.

Professores podem contribuir para a diminuição de situações eliciadoras dessas emoções, por exemplo, deixando de fazer e de comunicar julgamentos causais interpessoais que levem os alunos a desenvolver crenças atribuicionais desadaptadoras, às quais se

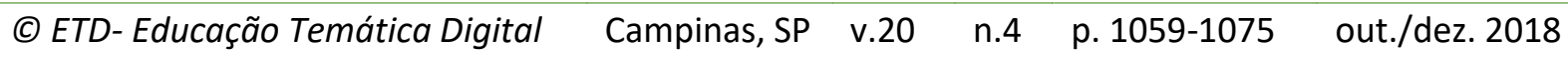


associam sentimentos de vergonha. Nas aulas, o tédio será combatido com argumentação reiterada sobre a importância das tarefas e dos conteúdos e com o uso de embelezamentos motivacionais. Por último, a evitação de toda forma de pressão em classe contribui para a prevenção de alta ansiedade nas situações de avaliação, com repercussão na fase de estudo (BZUNECK, 2016; BZUNECK; SALES, 2011; NETT et al., 2011; PEKRUN, 2006; VOGEL-WALKUTT et al., 2012; ZEIDNER, 2014).

Porém, apesar dos melhores esforços dos professores em seu ensino, sobram ocasiões em que caberá aos próprios alunos, de forma inteiramente solitária, a regulação de suas emoções. Primeiro, porque os professores em classe frequentemente não fazem sua parte de prevenção e até contribuem para ativar emoções negativas e, mesmo quando os professores cumprem bem o seu papel, não conseguem atingir todos os alunos, por razões diversas. Em segundo lugar, tédio e ansiedade assomam frequentemente no estudo individual, na preparação para provas ou no cumprimento do dever de casa, ou seja, na ausência de professores. Dessa forma, com base na literatura contemporânea, será agora demonstrado como, em qualquer contexto, alunos podem autorregular três emoções negativas, selecionadas por figurarem entre as mais recorrentes e que têm sido examinadas quanto à regulação: vergonha, tédio e ansiedade.

Autorregulação da vergonha/humilhação.

$\mathrm{Na}$ literatura foram relatadas duas linhas de atuação. A primeira, sugerida pela teoria de Weiner, que Zimmerman (2013) considerou, consiste no retreinamento das atribuições causais, uma estratégia muito eficaz que consiste em levar o aluno a atribuir qualquer resultado insatisfatório a fatores controláveis, como falta de esforço ou de uso de estratégias mais eficazes (por ex., HALL et al., 2007). Desta forma, atinge-se a raiz causadora da vergonha/humilhação, que fora a atribuição causal do mau resultado a algum fator interno não controlável.

Uma segunda linha de ação é sugerida pelos resultados do estudo de Turner et al. (2002), que identificaram dois tipos de alunos em relação à emoção de vergonha: os resilientes e os não-resilientes. Os primeiros caracterizavam-se por grau acentuado de motivação extrínseca, senso de competência e estabelecimento de metas futuras. Já os nãoresilientes mostravam-se mais confusos ou não definidos em qualquer dessas três variáveis motivacionais. Em particular, não viam clara relação entre o que deveriam fazer para atingir objetivos de vida, ou seja, faltava-Ihes a percepção de que metas futuras podem ser atingidas com persistente uso de estratégias adequadas, que revelaram desconhecer. A recuperação das crenças de autoeficácia será fator crítico, porém, segundo as mesmas autoras, é processo que demanda mais tempo.

A implicação clara desses dados é que alunos têm capacidade de superar seu sentimento de vergonha, com a condição de que exerçam autorregulação de processos motivacionais, que incluem a crença de autoeficácia. Assim, em primeiro lugar, exige-se que 
o aluno tome consciência de que sua motivação se encontra abalada por desacreditar de sua capacidade, ou seja, porque sua autoeficácia está reduzida (WOLTERS, 2011). Como primeira ação proativa destinada a recuperar a autoeficácia, é sugerida a introdução de um diálogo interno do tipo "eu sei que posso realizar essa tarefa", como foi sugerido por Bandura (1997). Para ser eficaz, porém, a verbalização deve ter apoio na evocação de situações passadas de superação de dificuldades, ou seja, experiências de domínio. Em particular, também contribui muito a verificação do próprio progresso, uma vez que toda percepção de progresso, contem mensagem alimentadora de autoeficácia. Com essas estratégias, há boas expectativas de resiliência, ou seja, que o aluno não somente recupere a crença de autoeficácia como alimente a crença de que bons resultados acadêmicos dependem mais de esforço, com boas estratégias, fatores que estão inteiramente em suas mãos.

\section{Autorregulação do tédio.}

Nett et al. (2010) e Nett et al. (2011) propuseram que o meio mais eficaz de o próprio aluno superar tédio, seja enquanto traço/disposição ou como estado, consiste em alimentar a percepção de valor da atividade, em termos de sua utilidade ou como importante. Trata-se de uma estratégia cognitivo-aproximativa, de cunho motivacional, que atinge a raiz do tédio. É cognitiva, porque aí está envolvido um processo de reavaliação ou mudança de pensamento a respeito da situação. E é aproximativa porque se trata de uma estratégia com a qual o problema é diretamente enfrentado, o que sugere proatividade por parte do aluno. $O$ valor instrumental será ativado quando se passar a considerar conscientemente que o engajamento em certa atividade de aprendizagem e até mesmo a participação ativa em classe são úteis para se atingirem objetivos mais distantes que o aluno tenha em mente, como fazer novos cursos, conseguir uma boa profissão futura, ou para objetivos próximos como conseguir boas notas ou ser reconhecido. Essa estratégia mostrou-se eficaz não apenas para controle do tédio já instalado, mas também como medida de prevenção.

Adicionalmente, poderá contribuir para o controle pessoal do tédio a estratégia de reativar a própria motivação intrínseca, pela autopromoção de interesse por uma tarefa que não apareça como de importância pessoal (PAULINO et al., 2015; WOLTERS, 2003). A sugestão desses autores é transformá-la numa forma de jogo, o que se estima ser viável em certas tarefas de matemática. Em suma, já que tédio em situações acadêmicas tem raiz numa falta de percepção de valor ou no desinteresse pela atividade, estratégias que incidem sobre esses fatores atacarão a própria raiz da emoção negativa.

\section{Autorregulação da ansiedade.}

Como a alta ansiedade, seja em situação de provas, seja em relação a alguma disciplina como matemática tem como componentes indissociáveis a emotividade e o aspecto cognitivo de preocupação, a maioria dos pesquisares tem proposto que a autorregulação deva atingir

\begin{tabular}{|c|c|}
\hline (C) ETD- Educação Temática Digital & Camninas SP y 20 n. 4 o $1059-1075$ \\
\hline
\end{tabular}


ambos os componentes (por ex., AHMED et al., 2013; HEMBREE, 1988; HARPELL; ANDREWS, 2013; ZEIDNER, 2014), porém, com mais ênfase sobre o cognitivo. Como síntese das revisões de estudos de intervenção empreendidos por esses e outros autores, técnicas de dessensibilização e relaxamento têm sido eficazes para reduzir a emotividade e até melhorar o rendimento. Além disso, melhores resultados advieram de técnicas que focalizavam a emotividade combinadas com técnicas de tipo cognitivo, que incluíam treino da atenção e modificação cognitiva. Esta última modalidade consiste em o aluno produzir outro diálogo interno construtivo, que substitua o componente cognitivo de preocupação (ZEIDNER, 2014). Na mesma linha, também Pekrun (2006), que havia adotado o conceito de emoções habitualizadas, propôs que a redução de emoções negativas, como ansiedade, deve ser procurada por meio de mudanças na avaliação da situação ansiógena, ou seja, pela quebra dos esquemas mentais de procedimento mantidos pelo aluno, com os quais ele associa certo ambiente com alta ansiedade.

Segundo Dembo (2000), a modificação cognitiva pressupõe que o aluno identifique, por automonitoramento, o diálogo interno intruso, em termos de preocupações, que o acomete, podendo ser feito até por escrito. A escala construída por Pekrun et al. (2011) fornece sugestões de como aplicar tal monitoramento, após o qual o aluno será capaz de intervir eficazmente com um diálogo interno alternativo, que será em termos positivos e que dê apoio.

Como primeira orientação, Dembo (2000) advertiu serem ineficazes autocomandos na forma negativa, do tipo "não vou entrar em pânico quando começar a prova" ou com uma dúvida, "mas se...". Na mesma linha, DeCuir-Gumbi et al. (2009) descobriram que, na hora da prova, estratégias de autocensurar-se ou de acalentar a ideia ilusória de que a realidade podia ser diferente (dizendo, por exemplo, para si mesmo: tomara que o professor anule esta prova...Ah! se eu pudesse sair da sala agora!...) não somente eram inócuas como tinham relação positiva e significativa com irritação e com a própria ansiedade de prova. Logo, são formas de diálogo interno contraproducente.

Diálogo interno eficaz consistirá de frases diferentes, como estas: "Eu estou preparado para esta prova", "eu posso dar conta disso", "com a prática, tenho conseguido..." e, caso estiver bloqueado numa questão, dirá: "vou passar para outra, depois eu volto" (JACOBS; GROSS, 2014; PINTRICH, 2004; ZEIDNER, 2014). Além da linguagem interiorizada, imediatamente antes de uma prova importante, uma breve redação escrita na qual se expressem as preocupações em relação à prova, promovem melhora no desempenho, como foi descoberto em estudos experimentais (RAMIREZ; BEILOCK, 2011).

Outra estratégia cognitiva eficaz consiste em o aluno buscar controlar a própria atenção, direcionando-a para o teor de uma questão da prova, o que contribuirá para afastar os pensamentos espúrios (DECUIR-GUMBI et al., 2009; HEMBREE, 1988; JACOBS; GROSS, 2014). Muitos alunos têm, rotineiramente, dificuldades com os processos da atenção. Justicia-Galiano et al. (2016) haviam descoberto que universitários já sofriam de certa

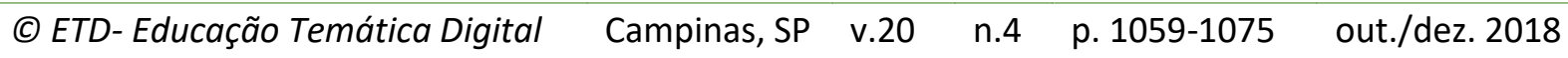


tendência à dispersão mental até em atividades diárias, com fácil reocorrência nas aulas e em situações de provas. Alunos dessa categoria carecerão de mais treinamento destinado a melhorar os processos de atenção.

Na mesma linha, DeCuir-Gunbi et al. (2009) relataram que a estratégia de respirar fundo ou de parar por um minuto pode ajudar a reduzir os pensamentos espúrios do diálogo interno. Além disso, estudos citados por Jacobs e Gross (2014) mostraram que também é eficaz a técnica cognitiva de reinterpretar a emocionalidade inerente à ansiedade de prova, passando a considerá-la como respostas positivas e benéficas, ou atribuindo-a a fontes irrelevantes, em vez de creditá-las à própria situação de prova.

\section{Interpretação à luz da Teoria Social Cognitiva}

A Teoria social cognitiva (BANDURA, 1997; 2006) tem sido apresentada como referencial quando se trata da regulação das emoções, uma vez que fornece argumentação de fundo sobre porque regular emoções, enfatizando sua ligação íntima com aprendizagem e motivação, além de proporcionar uma interpretação dos resultados de forma coerente com o contexto educacional. Segundo Bandura (2006), Pintrich (2004) e Zimmerman (2013), os alunos também podem regular seus afetos, da mesma forma como podem regular suas cognições, sua motivação e seus comportamentos, bem como o próprio ambiente. Porém, raramente ou nunca, os estudos sobre regulação das emoções tiveram como referencial a Teoria social cognitiva ou os dados foram interpretados à luz dessa teoria. Entretanto, é ela que proporciona elementos mais completos para se interpretarem os resultados obtidos até o presente. A adoção da Teoria social cognitiva permitirá um avanço significativo nos estudos sobre as emoções na educação que, para Mega et al. (2014), ainda se apresentam relativamente fragmentados, faltando melhor integração conceitual sobre a própria emoção e sua relação com cognição e motivação.

O conceito de autorregulação está aqui intimamente atrelado ao de agência e relacionado aos comportamentos direcionados para objetivos de aprendizagem escolar. Segundo Bandura (1997), trata-se da condição em que pessoas (em nosso caso, alunos) podem monitorar e exercer influência sobre o que elas fazem, pensam e sentem, assim como sobre o ambiente, sem esquecer que a relação é recíproca. Aqui entra a característica da autorreatividade, isto é, ao avaliar uma situação como geradora de emoções negativas, o aluno exercerá autorregulação sobre elas, estabelecendo-a como uma meta próxima a atingir. Zimmerman (2013) chamou de proativos os alunos que adotam tais estratégias de autorregulação, ao contrário dos reativos, que deixam de executar tais processos, ficando então sujeitos aos possíveis efeitos perversos das emoções negativas e, na melhor das hipóteses, dependentes de condições ambientais favoráveis.

\section{O desenvolvimento da autorregulação emocional}

Ahmed et al. (2013) relataram que pouco se sabe sobre os processos de desenvolvimento das emoções nas trajetórias escolares dos alunos. Mais ainda, parece não

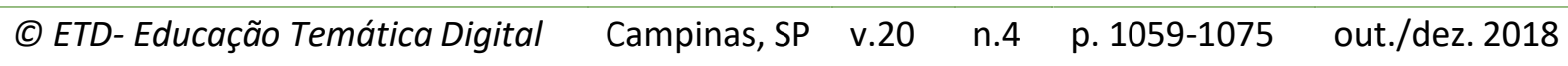


ter havido a preocupação de demonstrar como, à luz de uma teoria, se tem chegado a ajudar alunos a controlarem emoções negativas que porventura sofram de modo crônico, ou seja, a desenvolverem a habilidade de aurorregulação emocional. Nesse sentido, professores passarão a seus alunos a mensagem de que, em muitos casos, a eles caberá o papel de regular suas próprias emoções no contexto acadêmico.

Mesmo que inicialmente motivado para aprender, um aluno terá ainda, eventualmente, que desenvolver a autorregulação de suas emoções negativas. Embora alguns já o consigam, dificilmente pode-se esperar que todos os alunos disponham de conhecimento tanto declarativo como procedimental e condicional das estratégias de autorregulação das diversas emoções negativas. Por exemplo, como se pode supor que eles saibam como superar o tédio, sem receber ajuda externa que demonstre como superá-lo? 0 mesmo vale para a ansiedade: como os alunos saberão produzir um diálogo interno positivo, que substitua o prejudicial, pela quebra dos esquemas mentais associados à ansiedade em certas situações? Como controlar os processos de atenção nas provas? Quanto à experiência de vergonha/humilhação, alunos saberão mudar as atribuições disfuncionais para outras mais produtivas? Em qualquer desses casos, alguns alunos até poderiam chegar a conhecer as habilidades exigidas, com a ajuda de textos específicos e bem fundamentados, caso Ihes sejam acessíveis. Assim mesmo, faltaria treinamento monitorado. Entretanto, um procedimento, inspirado na teoria social cognitiva e descrito por Zimmerman (2013), com base em estudos anteriores, como o de Schunk e Zimmerman (1997), tem alcance generalizado.

Zimmermann (2013) apresentou um modelo de quatro etapas pelas quais um aluno poderá desenvolver a autorregulação da aprendizagem, havendo razões plausíveis de que seja igualmente aplicável à dimensão específica da autorregulação das emoções negativas. Função crucial foi atribuída a fatores sociais, pois, como defendeu Bandura (1997), não se pode prescindir da influência desses fatores quando se trata de esforços para a autorregulação durante as aprendizagens. Assim, o fator básico e imprescindível, segundo o modelo, consiste no processo de modelação, pelo qual o aluno será exposto a modelos de reconhecimento e de monitoramento dos estados afetivos negativos, bem como das diversas estratégias destinadas à sua autorregulação. Isto é, pessoas significativas como professores, colegas ou pais darão o modelo de como identificar e enfrentar cada uma das emoções negativas, demonstrando como se executa cada estratégia relatada como potencialmente eficaz. Em suma, o modelo de autorregulação influenciará duplamente. Primeiro, porque apresenta o padrão cognitivo-comportamental de como proceder nos casos de emoções prejudiciais identificadas. Segundo, porque o modelo de uso da estratégia bem-sucedida também é fonte de autoeficácia (BANDURA, 1997), fator motivacional crítico para a autorregulação.

Após essa fase de modelação, de acordo com Zimmerman (2013), virá a de emulação, em que o aluno mostrará experiências de aplicação em novas tarefas dos padrões aprendidos,

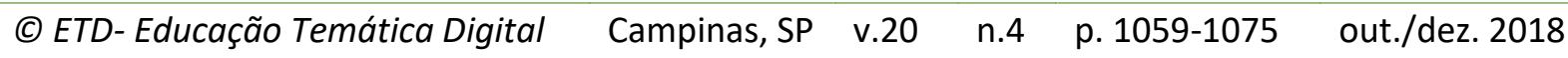


mas caberia ainda certa orientação, apoio e feedback dados por pessoas competentes. A terceira fase é a do autocontrole, em que o aluno praticará extensivamente e por própria iniciativa as habilidades exigidas, mas sem a presença do modelo. Autopercepção de êxito será a recompensa pelos esforços. O nível autorregulado é a etapa final, quando o aluno for capaz de adaptar, de modo sistemático e autônomo, conforme exigências pessoais e situacionais cambiantes, os conhecimentos e as habilidades adquiridas. Aí atuarão como motivadores a orientação as metas e, sobretudo, as crenças de autoeficácia, que seguirão alimentadas pela constatação de resultados bem-sucedidos.

\section{SUGESTÕES PARA PESQUISAS FUTURAS}

Para o contexto educativo em nosso meio, dada a escassez de pesquisas nessa área, abrem-se várias linhas de investigação, particularmente em termos de intervenção remediadora, tomando-se como referencial a Teoria Social Cognitiva. Em função das diferenças individuais entre alunos e da grande variedade de situações potencialmente estressantes na escola, a investigação sobre maneiras de ajudar os alunos a desenvolverem autorregulação das emoções negativas deverá atender a situações mais restritas. Por exemplo, tédio e ansiedade são emoções passíveis de serem investigados não apenas em relação a uma disciplina ou professor, às situações de prova ou ao cumprimento de dever de casa, como também em relação a segmentos de uma aula, como fazer exercícios, envolverse em discussão ou em trabalho em grupo.

Além disso, pouco ou nada se sabe sobre os efeitos de cada estratégia específica de regulação, dadas as diferenças entre os alunos e os níveis de escolaridade. Isto é, qual estratégia funcionará melhor com determinado estudante? E, por último, o que não é menos importante, resta ainda investigar como alunos poderiam, nos contextos de aprendizagem, regular e explorar construtivamente emoções como frustração e irritação, além de emoções positivas como orgulho, alívio, satisfação e esperança.

\section{REFERÊNCIAS}

AHMED, W.; VAN DER WERF, G.; KUYPER, H.; MINNAERT, A. Emotions, self-regulated learning, and achievement in mathematics: a growth curve analysis. Journal of Educational Psychology, v. 105, n. 1, p. 150-161, 2013.

ASHBY, F. G.; ISEN, A. M.; TURKEN, A. U. A neuropsychological theory of positive affect and its influence on cognition. Psychological Review, v. 106, p. 529-550, 1999.

BANDURA, A. Self-efficacy: the exercise of control. New York: W.H. Freeman and Company, 1997.

BANDURA, A. Adolescent development from an agentic perspective. In: PAJARES, F.; URDAN, T. (Ed.), Self-efficacy beliefs of adolescents. Greenwich, CT: Information Age, 2006, p.1-43. 
BEN-ELIYAHU, A.; LINNENBRINK-GARCIA, L. Integrating the regulation of affect, behavior, and cognition into self-regulated learning paradigms among secondary and postsecondary students. Metacognition and learning, v. 10, n. 1, p. 15-42, 2015.

BZUNECK, J. A. Ansiedade e desempenho numa prova de matemática: um estudo com adolescentes. Revista Semina (Londrina), v. 12, n. 3, p. 136-141, 1991.

BZUNECK, J.A. Como motivar os alunos: sugestões práticas. In: BORUCHOVITCH, E.; BZUNECK, J.A.; GUIMARÃES, S.E.R. Motivação para aprender: aplicações no contexto educativo. Petrópolis RJ: Vozes, 2016. p. 13-42.

BZUNECK, J.A.; SALES, K.F.S. Atribuições interpessoais pelo professor e sua relação com motivação e emoções do aluno. Psico-USF, v. 16, n. 3, p. 307-315, 2011.

CASSADY, J.C.; JOHNSON, R.E. Cognitive test anxiety and academic performance.

Contemporary Educational Psychology, v. 27, p. 270-295, 2002.

CSIKSZENTMIHALYI, M. A Psicologia da felicidade. São Paulo: Saraiva. 1992.

DAI, Y.D.; STERNBERG, R.J. Beyond cognitivism: toward an integrated understanding of intellectual functioning and development. In: DAI, D.Y.; STERNBERG, R.J. (Ed.) Motivation, emotion, and cognition. Mahwah, NJ: Lawrence Erlbaum, 2004, p. 3-38.

DANIELS, L. M.; STUPNISKY, R.H.; PEKRUN, R. ; HAYNES, T.L.; PERRY, R. P.; NEWAL, N.E. A longitudinal analysis of achievement goals: from affective antecedents to emotional effects and achievement outcomes. Journal of Educational Psychology, v. 101, n. 4, p. 948-963, 2009.

DECUIR-GUNBY, J.T.; AULTMAN, L.P.; SCHUTZ, P. A. Investigating transactions among motives, emotional regulation related to testing, and test emotions. The Journal of Experimental Education, v. 77, n. 4, p. 409-436, 2009.

$D E M B O, M . H$. Motivation and learning strategies for college success. A self-management approach. Mahwah, NJ: Lawrence Erlbaum, 2000.

ECKERT. M.; EBERT, D.D.; LEHR, D.; SIELAND, B.; BERKING, B. Overcome procrastination: enhancing emotion regulation skills reduce procrastination. Learning and Individual Differences, v. 52, p. 10-18, 2016.

ERTURAN, S.; JANSEN, B. An investigation of boys and girls' emotional experience of math, their math performance, and the relation between these variables. European Journal of Psychology of Education, v. 30, p. 421-435, 2015.

FIORAVANTI-BASTOS, A.C. M.; CHENIAUX, E.; LANDEIRA-FERNANDEZ, J. Development and validation of a short-form version of the brazilian state-trait anxiety inventory. Psicologia: Reflexão e Crítica, v. 24, n. 3, p. 485-494, 2011.

FREDERICKSON, B.L. The role of positive emotions in positive psychology: the broaden-andbuilt theory of positive emotions. American Psychologist, v. 56, p. 218-256, 2001.

HALL, N. C.; PERRY, R. P.; GOETZ, T.; RUTHIG, J. C.; STUPNISKY, R. H.; NEWALL, N. E. Attributional retraining and elaborative learning: Improving academic development through writing-based interventions. Learning and Individual Differences, v. 17, n. 3, p. 280-290, 2007. 
HARPELL, J.V.; ANDREWS, J.J.W. Relationship between school based stress and test anxiety. International Journal of Psychological Studies, v. 5, n. 2, p. 74-84, 2013.

HEMBREE, R. Correlates, causes, effects, and treatment of test anxiety. Review of Educational Research, v. 58, n. 1, p. 47-77, 1988.

HUANG, C. Achievement goals and achievement emotions: a meta-analysis. Educational Psychology Review, v. 23, p. 359-388, 2011.

JACOBS, S.E.; GROSS, J.J. Emotion regulation in education: conceptual foundations, current applications, and future directions. In: R. PEKRUN; L. LINNENBRINK-GARCIA (Ed.), International handbook of emotions in education. New York, NY: Routledge, 2014. p.183202.

JUSTICIA-GALIANO, M.-J.; PELEGRINA, S.; LECHUGA, M.-T.; GUTIÉRREZ-PALMA, N.; MARTÍNPUGA, E.-M.; LENDÍNEZ, C. Math anxiety and its relationship to inhibitory abilities and perceived emotional intelligence. Anales de Psicologia (Murcia), v. 32, n. 1, p. 125-131, 2016.

LIEBERT, R.N.; MORRIS, L.W. Cognitive and emotional components of test anxiety: a distinction and some initial data. Psychological Reports, v. 20, p. 975-978, 1967.

LINNENBRINK-GARCIA, L.; PEKRUN, R. Students' emotions and academic engagement: introduction to the special issue. Contemporary Educational Psychology, v, 36, p. 1-3, 2011. MEGA, C.; RONCONI, L.; DE BENI, R. What Makes a Good Student? How emotions, selfregulated learning, and motivation contribute to academic achievement. Journal of Educational Psychology, v. 106, n. 1, p. 121-131, 2014.

NETT, U. E.; GOETZ, T.; DANIELS, L. M. What to do when feeling bored? Students' strategies for coping with boredom. Learning and Individual Differences, v. 20, p. 626-638, 2010.

NETT, U. E.; GOETZ, T.; HALL, N. C. Coping with boredom in school: an experience sampling perspective. Contemporary Educational Psychology, v. 36, p. 49-59, 2011.

OECD. Organization for Economic Co-operation and Development. PISA 2012 Results in Focus. Paris: OECD, 2013.

OP'T EYNDE, P.; TURNER, J. E. focusing on the complexity of emotion issues in academic learning: a dynamical component systems approach. Educational Psychology Review, v.18, p. 361-376, 2006.

PAULINO, P.; SÁ, I.; SILVA, A. L. Autorregulação da motivação: crenças e estratégias de alunos portugueses do 70 ao 9ㅇa ano de escolaridade. Psychology /Psicologia Reflexão e Crítica, v. 28, n. 3, p. 574-582, 2015

PEKRUN, R. The control-value theory of achievement emotions: assumptions, corollaries, and implications for educational research and practice. Educational Psychology Review, v. 18, p. 315-341, 2006.

PEKRUN, R.; GOETZ, T.; TITZ, W.; PERRY, R.P. Academic emotions in students' self-regulated learning and achievement: a program of qualitative and quantitative research. Educational Psychologist, v. 3, p. 91-105, 2002. 
PEKRUN, R.; GOETZ, T.; FRENZEL, A. C.; BARCHFELD, P.; PERRY, R. P. Measuring emotions in students' learning and performance: the Achievement Emotions Questionnaire (AEQ). Contemporary Educational Psychology, v. 36, p. 36-48, 2011.

PEKRUN, R.; HALL, N. C.; GOETZ, T.; PERRY, R. P. Boredom and academic achievement: testing a model of reciprocal causation. Journal of Educational Psychology, v. 106, p. 696710, 2014.

PINTRICH, P.R. A conceptual framework for assessing motivation and self-regulated learning in college students. Educational Psychology Review, v. 16, n. 4, p. 385-406, 2004.

RAMIREZ, G.; BEILOCK, S. L. Writing about testing worries boosts exam performance in the classroom. Science, v. 331, p. 211-213, 2011.

REALI, F.; JIMÉNEZ-LEAL, W.; MALDONADO-CARREÑO, C. Examining the link between math anxiety and math performance in colombian students. Revista Colombiana de Psicología, v. 25, n. 2, p. 369-379, 2016.

SCHERER, K.R. The dynamic architecture of emotion: evidence for the component process model. Cognition and Emotion, v. 23, p. 1307-1351, 2009.

SCHUNK, D. H. Metacognition, Self-regulation, and self-regulated learning: research recommendations. Educational Psychology Review, v. 20, p. 463-467, 2008.

SCHUNK, D. H.; ZIMMERMAN, B. J. Social origins of self-regulatory competence. Educational Psychologist, v. 32, p. 195-208, 1997.

SPIELBERGER, C.D. Theory and research on anxiety. In: SPIELBERGER, C.D. (Ed.) Anxiety and behavior. New York: Academic Press, 1966. p. 3-20.

STEEL, $P$. The nature of procrastination: A meta-analytical and theoretical review of quintessential of self-regulation failure. Psycological Reports, v.133, p. 65-139, 2007.

TANAKA, A.; MURAYAMA, K. Within-person analyses of situational interest and boredom: interactions between task-specific perceptions and achievement goals. Journal of Educational Psychology, v. 106, n. 4, p. 1122-1134, 2014.

TURNER, J. E.; HUSMAN, J.; SHALLERT, D.L. The importance of student's goals in their emotional experience of academic failure: investigating the precursors and consequences of shame. Educational Psychologist, v. 37, p. 79-89, 2002.

TZE, V. M. C.; DANIELS, L.M.; KLASSEN, R.M. Evaluating relationships between boredom and academic outcomes. Educational Psychology Review, v. 28, n. 1, p. 119-144, 2016.

VIERHAUS, M.; LOHAUS , A.; WILD, E. The development of achievement emotions and coping/emotion regulation from primary to secondary school. Learning and Instruction, $v$. 42, apr., p. 12-21, 2016.

VOGEL-WALCUTT, J. J.; FIORELLA, L.; CARPER, T.; SCHATZ, S. The definition, assessment, and mitigation of state boredom within educational settings: a comprehensive review.

Educational Psychology Review, v. 24, p. 89-111, 2012

VOGELAAR, B.; BAKKER, M.; ELLIOT, J.G.; RESING, W.C.M. Dynamic testing and test-anxiety amongst gifted and average ability children. British Journal of Educational Psychology, 87, p.75-89, 2017. 
WEINER, B. An attributional theory of achievement motivation and emotion. Psychological Review, v. 92, p. 548-573, 1985.

WOLTERS, C.A. Regulation of motivation: evaluating an underemphasized aspect of selfregulated learning. Educational Psychologist, v. 38, n. 4, p. 189-205, 2003.

WOLTERS, C. Regulation of motivation: contextual and social aspects. Teachers College Record, v. 113, p. 265-283, 2011.

ZEIDNER, M. Anxiety in Education. In: R. PEKRUN; L. LINNENBRINK-GARCIA (Ed.), International Handbook of Emotions in Education. New York, NY: Routledge, 2014. p. 265288.

ZIMMERMAN, B. J. From cognitive modeling to self-regulation: a social cognitive career path. Educational Psychologist, n. 48, n.3, p. 135-147, 2013.

*Revisão gramatical do texto sob responsabilidade de:

Prof. Aluysio Fávaro

E-mail: jukerche@sercomtel.com.br 